\title{
COVERING MORPHISMS IN CATEGORIES OF RELATIONAL ALGEBRAS
}

\author{
MARIA MANUEL CLEMENTINO, DIRK HOFMANN, AND ANDREA MONTOLI
}

Dedicated to George Janelidze on his sixtieth birthday

\begin{abstract}
In this paper we use Janelidze's approach to the classical theory of topological coverings via categorical Galois theory to study coverings in categories of relational algebras. Moreover, we present characterizations of effective descent morphisms in the categories of $M$ ordered sets and of multi-ordered sets.
\end{abstract}

\section{INTRODUCTION}

Reiterman-Tholen characterization of effective descent morphisms in the category Top of topological spaces [23] showed the relevance of convergence in topological descent theory. This was further stressed in Janelidze-Sobral [20] approach to finite topological descent, and later used by Clementino and Hofmann in the infinite case [4]. Following a suggestion by Janelidze, Barr's contribution [1] on relational algebras - showing that topological spaces are lax Eilenberg-Moore algebras for the ultrafilter monad - was revisited (see $[5,13]$ ), and some results on topological descent theory were extended to this setting (see $[6,7]$ ).

Recently Janelidze raised the question whether the study of classical topological coverings via categorical Galois Theory (see $[2,15,16,18]$ ) could be extended to the setting of relational algebras. Indeed, when the category $\operatorname{Conn}(\mathbf{C})$ has a terminal object, the connected component functor has a right adjoint

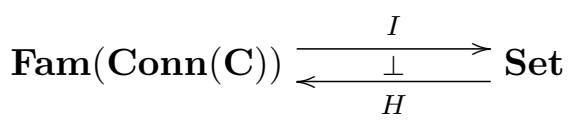

Since this adjunction is semi-left exact [3], this gives a Galois structure. For $\mathbf{C}=$ Top, in order to study coverings for this adjunction, it is necessary to consider its restriction to the category of locally connected spaces and local homeomorphisms. Then Galois coverings coincide with classical topological coverings. It is the purpose of this paper to give a first contribution to the study of coverings in the realm of relational algebras.

In the first section we recall basic results on relational $\mathbb{T}$-algebras, in particular some known results on descent, and obtain new characterizations for effective descent maps in the categories of $M$-ordered sets, for a given monoid $M$, and of multi-ordered sets.

In Section 2 we present Mahmoudi-Schubert-Tholen results [21] on coproducts and extensivity of the category of relational algebras, and study a functor $\mathcal{O}$ that assigns to each relational algebra a topology (also introduced in [21]). This functor $\mathcal{O}$ plays a key role in the study of local isomorphisms, as introduced here.

In Section 3 we recall the definition of connected object in an extensive category and study properties of connected relational algebras. Then, in Section 4, we show that the semi-left exact

Research partially supported by Centro de Matemática da Universidade de Coimbra, by Centro de Investigação e Desenvolvimento em Matemática e Aplicações da Universidade de Aveiro/FCT, by Fundação para a Ciência e a Tecnologia, through European program COMPETE/FEDER, via the projects MCANA and MONDRIAN (under the contracts PTDC/MAT/120222/2010 and PTDC/EIA-CCO/108302/2008), and the grant SFRH/BPD/69661/2010. 
connected component adjunction has stable units provided that the functor $T$ preserves the terminal object.

In Section 5 we study covering morphisms when $T$ preserves intersections, so that every relational $\mathbb{T}$-algebra is a coproduct of connected relational $\mathbb{T}$-algebras. For the categories of $M$-ordered sets and multi-ordered sets we give characterizations of covering maps.

Section 6 is devoted to the generalization of Janelidze's approach to topological coverings, as presented in $[2$, Chapter 6].

\section{Relational ALGebras And their efFeCtive DESCENT MORPhisms}

Let $\mathbb{T}=(T, \eta, \mu)$ be a monad on Set. We denote by $\mathbf{A} \lg (\mathbb{T})$ the category of Eilenberg-Moore $\mathbb{T}$-algebras; that is, an object of $\operatorname{Alg}(\mathbb{T})$ is a pair $(X, \alpha)$, where $X$ is a set and $\alpha: T X \rightarrow X$ is a map making the following diagram commutative

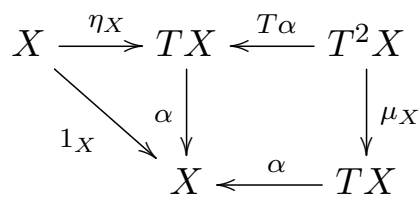

and a morphism $f:(X, \alpha) \rightarrow(Y, \beta)$ is a map $f: X \rightarrow Y$ with $f \cdot \alpha=\beta \cdot T f:$

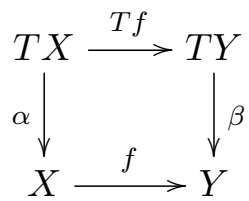

The functor $T$ defines an oplax functor $\bar{T}: \operatorname{Rel} \rightarrow \operatorname{Rel}\left(\right.$ see [1]), with $\overline{T r}=\operatorname{Tr}_{2} \cdot\left(\operatorname{Tr}_{1}\right)^{\circ}$, for any relation $r: X \mapsto Y$, with $r=r_{2} \cdot r_{1}^{\circ}$,

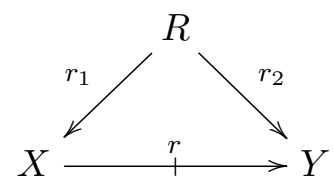

where $r_{1}, r_{2}$ are the projections and $r_{1}^{\circ}$ is the opposite relation of $r_{1} . \bar{T}: \mathbf{R e l} \rightarrow \mathbf{R e l}$ is a (strict) functor if and only if $T$ : Set $\rightarrow$ Set has the Beck-Chevalley property (BC) (in the sense of [5]), that is $T$ preserves $(\mathrm{BC})$-squares, where a $(B C)$-square is a diagram

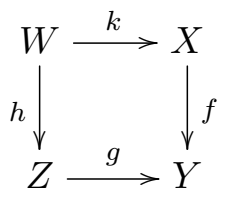

such that $f^{\circ} \cdot g=k \cdot h^{\circ}$, with $f^{\circ}$ and $h^{\circ}$ the opposite relations of $f$ and $h$, respectively. Every pullback preserving functor - that is, every cartesian functor - has (BC), and (BC) implies, in particular, that the functor preserves pullbacks along monomorphisms, that is, it is taut (see $[22,10])$.

Throughout we assume that $T:$ Set $\rightarrow$ Set has the $(B C)$ property. We will work on the category $\operatorname{RelAlg}(\mathbb{T})$ of relational $\mathbb{T}$-algebras (or lax algebras, or $(\mathbb{T}, 2)$-categories: see $[1,5,13]$ ), with objects pairs $(X, a)$, where $X$ is a set and $a: T X \rightarrow X$ is a relation such that $1_{X} \leq a \cdot \eta_{X}$ 
and $a \cdot \bar{T} a \leq a \cdot \mu_{X}$ :

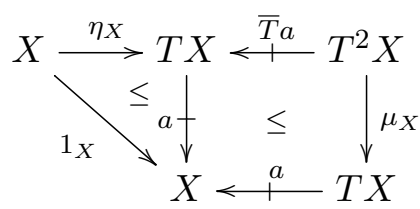

(These inequalities capture reflexivity and transitivity properties for $a$ in the sense below.) Morphisms $f:(X, a) \rightarrow(Y, b)$ between relational $\mathbb{T}$-algebras are maps $f: X \rightarrow Y$ with $f \cdot a \leq b \cdot T f:$

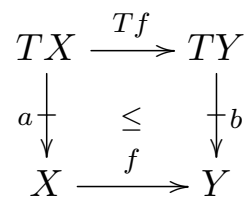

We will often use pointwise notation on relational algebras: for $x \in X$ and $\mathfrak{x} \in T X$, we will write $\mathfrak{x} \rightarrow x$ whenever $\mathfrak{x} a x$; a relation $a: T X \mapsto X$ is a relational $\mathbb{T}$-algebra if

$$
\begin{array}{ll}
\eta_{X}(x) \rightarrow x & (a \text { is reflexive }), \text { and } \\
\left(\mathfrak{X} \rightarrow \mathfrak{x} \& \mathfrak{x} \rightarrow x \Rightarrow \mu_{X}(\mathfrak{X}) \rightarrow x\right) & (a \text { is transitive }),
\end{array}
$$

for every $x \in X, \mathfrak{x} \in T X$ and $\mathfrak{X} \in T T X$ (here $\mathfrak{X} \rightarrow \mathfrak{x}$ means $\mathfrak{X}(\bar{T} a) \mathfrak{x}$ ); a map $f: X \rightarrow Y$ is a morphism $f:(X, a) \rightarrow(Y, b)$ if

$$
\mathfrak{x} \rightarrow x \Rightarrow T f(\mathfrak{x}) \rightarrow f(x),
$$

for every $x \in X, \mathfrak{x} \in T X$.

By a pseudo-relational $\mathbb{T}$-algebra we mean a reflexive relation $a: T X \rightarrow X$, so that $\eta_{X}(x) \rightarrow x$ for every $x \in X$; a morphism $f:(X, a) \rightarrow(Y, b)$ between pseudo-relational $\mathbb{T}$-algebras is a map $f: X \rightarrow Y$ with $T f(\mathfrak{x}) \rightarrow f(x)$ whenever $\mathfrak{x} \rightarrow x$, for $x \in X$ and $\mathfrak{x} \in T X$. We denote the category of pseudo-relational $\mathbb{T}$-algebras by PsRelAlg $(\mathbb{T})$. We have the following commutative diagram

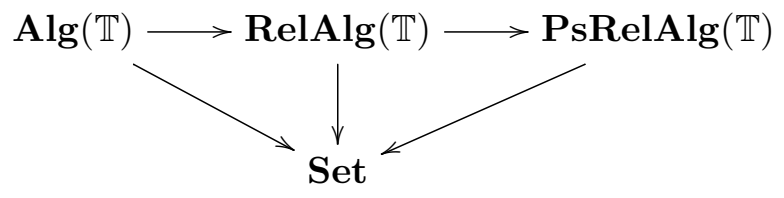

where the horizontal functors are full reflective embeddings and the vertical ones are forgetful functors. The functor $\mathbf{A l g}(\mathbb{T}) \rightarrow$ Set is monadic, while $\operatorname{RelAlg}(\mathbb{T}) \rightarrow \operatorname{Set}$ and PsRelAlg $(\mathbb{T}) \rightarrow$ Set are topological (see [5] for details). In particular, $\operatorname{RelAlg}(\mathbb{T})$ and $\operatorname{PsRelAlg}(\mathbb{T})$ are complete and cocomplete, and their forgetful functors preserve limits and colimits.

Given a relational algebra $(Y, b)$ and a map $f: X \rightarrow Y$, the initial structure $a$ on $X$ is given by $a=f^{\circ} \cdot b \cdot T f$ :

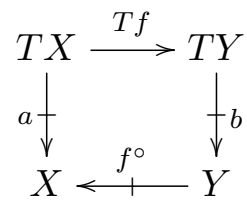

that is, for $x \in X, \mathfrak{x} \in T X$,

$$
\mathfrak{x} \rightarrow x \Leftrightarrow T f(\mathfrak{x}) \rightarrow f(x) .
$$

When $f:(X, a) \rightarrow(Y, b)$ is injective and $a$ is the initial structure for $f, f$ is said to be an embedding. 
If $f:(X, a) \rightarrow(Y, b)$ and $g:(Z, c) \rightarrow(Y, b)$ are morphisms in $\operatorname{RelAlg}(\mathbb{T})$, their pullback is built like in Set,

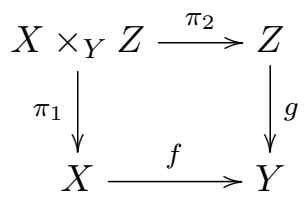

and the relational structure on $X \times_{Y} Z=\{(x, z) \in X \times Z \mid f(x)=g(z)\}$ is defined by

$$
\mathfrak{w} \rightarrow(x, z) \Leftrightarrow T \pi_{1}(\mathfrak{w}) \rightarrow x \text { and } T \pi_{2}(\mathfrak{w}) \rightarrow z,
$$

for any $\mathfrak{w} \in T\left(X \times_{Y} Z\right)$ and $(x, z) \in X \times_{Y} Z$.

Contrarily to $\operatorname{RelAlg}(\mathbb{T})$, which in general is not cartesian closed, PsRelAlg( $(\mathbb{T})$ is locally cartesian closed, as shown in [11]. As in every locally cartesian closed category, regular epimorphisms are pullback stable in PsRelAlg $(\mathbb{T})$, and they are in fact effective descent morphisms (descent morphisms), in the sense that their change-of-base functor is (pre)monadic (see [19]).

Proposition 1.1. For a morphism $f:(X, a) \rightarrow(Y, b)$ in PsRelAlg $(\mathbb{T})$, the following conditions are equivalent:

(i) $f$ is a regular epimorphism;

(ii) $f$ is stably a regular epimorphism;

(iii) $f$ is final, that is $b=f \cdot a \cdot(T f)^{\circ}$;

(iv) the change-of-base functor $f^{*}$ is premonadic;

(v) $f^{*}$ is monadic.

Here we recall some results on effective descent morphisms in $\operatorname{RelAlg}(\mathbb{T})$ we need throughout. Their proof relies on the embedding $\operatorname{RelAlg}(\mathbb{T}) \rightarrow \operatorname{PsRelAlg}(\mathbb{T})$ and on the behaviour of effective descent morphisms in PsRelAlg( $(\mathbb{T})$, as outlined in the following criterion, that follows from a well-known result of Descent Theory (see, e.g., [6, Theorem 2.1]).

Lemma 1.2. For a final morphism $f:(X, a) \rightarrow(Y, b)$ in $\operatorname{RelAlg}(\mathbb{T}), f$ is of effective descent if, and only if, for any morphism $g:(Z, c) \rightarrow(Y, b)$ in PsRelAlg $(\mathbb{T})$, if in the pullback (B) $X \times_{Y} Z$ is a relational $\mathbb{T}$-algebra, then $(Z, c)$ is a relational $\mathbb{T}$-algebra as well.

We say that the natural transformation $\eta$ has $(B C)$ for relations with finite fibres if every naturality square

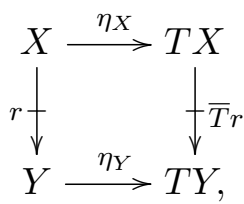

with $r$ a relation with finite fibres (so that $\{x \in X \mid x r y\}$ is finite for every $y \in Y$ ), is a (BC)-square, that is $(\bar{T} r)^{\circ} \cdot \eta_{Y}=\eta_{X} \cdot r^{\circ}$.

Theorem 1.3. [7, Theorem 2.4] If $\eta$ has (BC) for relations with finite fibres, then the following conditions are equivalent, for a morphism $f:(X, a) \rightarrow(Y, b)$ in $\operatorname{Rel} \mathbf{A l g}(\mathbb{T})$ :

(i) $f$ is final;

(ii) $f$ is a pullback stable regular epimorphism in $\operatorname{Rel} \mathbf{A l g}(\mathbb{T})$;

(iii) $f$ is a descent morphism in $\operatorname{RelAlg}(\mathbb{T})$. 
We remark that $f:(X, a) \rightarrow(Y, b)$ is final if and only if, for every $\mathfrak{y} \in T Y$ and $y \in Y$ with $\mathfrak{y} \rightarrow y$, there exist $\mathfrak{x} \in(T f)^{-1}(\mathfrak{y})$ and $x \in f^{-1}(y)$ such that $\mathfrak{x} \rightarrow x$. To guarantee that the morphism $f$ is of effective descent we will use a stronger property, called $*$-quotient morphism (following [23]): for each $\mathfrak{Y} \in T^{2} Y, \mathfrak{y} \in T Y$ and $y \in Y$, if $\mathfrak{Y} \rightarrow \mathfrak{y} \rightarrow y$ there exist $\mathfrak{X} \in\left(T^{2} f\right)^{-1}(\mathfrak{Y})$, $\mathfrak{x} \in(T f)^{-1}(\mathfrak{y})$ and $x \in f^{-1}(y)$ such that $\mathfrak{X} \rightarrow \mathfrak{x} \rightarrow x$.

Theorem 1.4. [6, Theorem 3.3] A morphism $f:(X, a) \rightarrow(Y, b)$ is an effective descent morphism in $\operatorname{RelAlg}(\mathbb{T})$ provided that it is a pullback stable $*$-quotient morphism in $\operatorname{PsRelAlg}(\mathbb{T})$.

It was an open question whether to be of effective descent would imply to be a $*$-quotient map. In Example 1.5.IV we solve the problem constructing an effective descent morphism which is not a $*$-quotient morphism.

\section{Examples 1.5.}

I. (Pre)Ordered sets. If $\mathbb{I}$ is the identity monad, then $\mathbf{A l g}(\mathbb{I})=$ Set and $\operatorname{RelAlg}(\mathbb{I})=\operatorname{Ord}$ is the category of (pre)ordered sets and monotone maps. (Here we do not assume antisymmetry of the order, just reflexivity and transitivity.)

Effective descent maps in Ord coincide with *-quotient maps (see [20]), that is a monotone map $f: X \rightarrow Y$ in Ord is of effective descent if, and only if,

$$
\left(\forall y_{0}, y_{1}, y_{2} \in Y\right): y_{2} \leq y_{1} \leq y_{0}\left(\exists x_{0} \in f^{-1}\left(y_{0}\right), x_{1} \in f^{-1}\left(y_{1}\right), x_{2} \in f^{-1}\left(y_{2}\right)\right): x_{2} \leq x_{1} \leq x_{0} .
$$

II. Topological spaces. If $\mathbb{U}$ is the ultrafilter monad, then $\mathbf{A l g}(\mathbb{U})$ is the category of compact Hausdorff spaces, RelAlg $(\mathbb{U})=$ Top is the category of topological spaces and PsRelAlg $(\mathbb{T})$ = PsTop is the category of pseudotopological spaces (and continuous maps).

Effective descent maps in Top were characterized in [23] via a elaborate convergence lifting property, that later, in [4], was translated to an ultrafilter convergence lifting, stated using an auxiliary functor Ult. A characterization using only the ultrafilter functor was obtained recently (see [12, Theorem 3.3]):

Theorem 1.6. For a continuous map $f: X \rightarrow Y$ between topological spaces, the following conditions are equivalent:

(i) $f$ is of effective descent in Top;

(ii) $f$ is stably a *-quotient map in PsTop.

We remark that it is not known whether in (ii) one can replace PsTop by Top.

III. $M$-ordered sets. If $\mathbb{M}=\left(M \times-, \eta_{X}=\left\langle e, 1_{X}\right\rangle, \mu_{X}=m \times 1_{X}\right)$, for a given monoid $(M, e, m)$, then $\operatorname{Alg}(\mathbb{M})=M$-Set is the category of $M$-sets while $\operatorname{RelAlg}(\mathbb{M})$ is the category $M$-Ord of $M$-labelled (reflexive and transitive) ordered sets, or simply $M$-ordered sets, and equivariant maps, when one thinks of $(m, x) \rightarrow y$ as a 'labelled order relation' $x \stackrel{m}{\longrightarrow} y$. In this category effective descent and $*$-quotient morphisms coincide, as we show next.

Lemma 1.7. In M-Ord *-quotient morphisms are pullback-stable.

Proof. Straightforward.

Theorem 1.8. An equivariant map $f:(X, a) \rightarrow(Y, b)$ in $M$-Ord is of effective descent if and only if it is a *-quotient map. 
Proof. The Lemma above, together with Theorem 1.4, gives that $*$-quotient maps are of effective descent. To show the converse, we assume that $f:(X, a) \rightarrow(Y, b)$ is an effective descent morphism and consider a chain $\gamma$ given by $\left(m, n, y_{2}\right) \rightarrow\left(m, y_{1}\right) \rightarrow y_{0}$ in $Y$. Define the pseudorelational structure $3_{\gamma}$ on $\{0,1,2\}$ with non-trivial relations $(k, i) \rightarrow j$ if $k=m, i=1, j=0$, or $k=n, i=2, j=1$. Let $g: 3_{\gamma} \rightarrow Y$ with $g(i)=y_{i}$. In the pullback of $f$ along $g$, $P:=(X, a) \times_{(Y, b)} 3_{\gamma}=\left\{(x, i) \mid f(x)=y_{i}, i=0,1,2\right\}$ cannot be an $M$-ordered set, due to Lemma 1.2. This implies that there exist $m^{\prime}, n^{\prime} \in M,\left(x_{k}, i_{k}\right) \in P, k=0,1,2$, such that $\left(m^{\prime}, x_{2}, i_{2}\right) \rightarrow\left(x_{1}, i_{1}\right),\left(n^{\prime}, x_{1}, i_{1}\right) \rightarrow\left(x_{0}, i_{0}\right)$ and $\left(m^{\prime} n^{\prime}, x_{2}, i_{2}\right) \not \rightarrow\left(x_{0}, i_{0}\right)$. Then necessarily $m^{\prime}=m, i_{2}=2$ and $n^{\prime}=n$ (or a permutation of this), and so there exist $x_{k} \in f^{-1}\left(y_{k}\right)$, $k=0,1,2$, such that $\left(m, n, x_{2}\right) \rightarrow\left(m, x_{1}\right) \rightarrow x_{0}$, that is $f$ is a $*$-quotient map.

We point out that, for $M=\{e\}$, this result gives the characterization in Ord we stated above.

IV. Multi-ordered sets. If $\mathbb{W}=(W, \eta, \mu)$ is the free monoid monad, then $\mathbf{A} \lg (\mathbb{W})$ is the category of monoids and $\operatorname{RelAlg}(\mathbb{W})$ is the category MultiOrd of multi-ordered sets and monotone maps. In this category effective descent morphisms are characterized by a lifting property of convergence that is weaker than the condition for $*$-quotient maps.

Lemma 1.9. For a pseudo-relational $\mathbb{W}$-algebra $(X, a)$, the following conditions are equivalent:

(i) $(X, a)$ is a multi-ordered set;

(ii) for every $x_{0}, x_{1}, \ldots, x_{n}, x_{1}^{i}, \ldots, x_{m}^{i} \in X$, with $i \in\{1, \ldots, n\}$, if $\left\langle x_{1}^{i}, \ldots, x_{m}^{i}\right\rangle \rightarrow x_{i}$ and $\left\langle x_{1}, \ldots, x_{n}\right\rangle \rightarrow x_{0}$, then $\left\langle x_{1}, \ldots, x_{i-1}, x_{1}^{i}, \ldots, x_{m}^{i}, x_{i+1}, \ldots, x_{n}\right\rangle \rightarrow x_{0}$.

Proof. Condition (ii) is a weak form of transitivity, hence (i) $\Rightarrow$ (ii).

(ii) $\Rightarrow$ (i): If $\left\langle\left\langle x_{1}^{1}, \ldots, x_{m_{1}}^{1}\right\rangle, \ldots,\left\langle x_{1}^{n}, \ldots, x_{m_{n}}^{n}\right\rangle\right\rangle \rightarrow\left\langle x_{1}, \ldots, x_{n}\right\rangle \rightarrow x_{0}$, then, for every $i=$ $1, \ldots, n,\left\langle x_{1}^{i}, \ldots, x_{n_{i}}^{i}\right\rangle \rightarrow x_{i}$. Considering $i=1$, we can apply (ii) to conclude that

$$
\left\langle x_{1}^{1}, \ldots, x_{m_{1}}^{1}, x_{2}, \ldots, x_{n}\right\rangle \rightarrow x_{0},
$$

and repeat successively this process for $i=2, \ldots, n$ to conclude that $(X, a)$ is transitive.

We say that a monotone map $f:(X, a) \rightarrow(Y, b)$ in PsRelAlg(W) is a weak $*$-quotient map if

(WQ) for each $y_{0}, y_{1}, \ldots, y_{n}, y_{1}^{i}, \ldots, y_{m}^{i} \in Y, i \in\{1, \ldots, n\}$, such that $\left\langle y_{1}^{i}, \ldots, y_{m}^{i}\right\rangle \rightarrow y_{i}$ and $\left\langle y_{1}, \ldots, y_{n}\right\rangle \rightarrow y_{0}$, there exist $x_{j} \in f^{-1}\left(y_{j}\right), j=1, \ldots, n$, and $x_{j}^{i} \in f^{-1}\left(y_{j}^{i}\right), j=1, \ldots, m$, such that $\left\langle x_{1}^{i}, \ldots, x_{m}^{i}\right\rangle \rightarrow x_{i}$ and $\left\langle x_{1}, \ldots, x_{n}\right\rangle \rightarrow x_{0}$.

It is clear that every weak $*$-quotient monotone map between multi-ordered sets is final, hence a descent morphism in MultiOrd by Theorem 1.3, since the natural transformation $\eta$ is cartesian.

Theorem 1.10. A monotone map is of effective descent in MultiOrd if and only if it is a weak *-quotient map.

Proof. It is easy to check that weak *-quotient maps are pullback-stable. By Lemma 1.2, to show they are of effective descent it is enough to show that transitivity descends along them, which is also straightforward, making use of the previous lemma.

To prove the converse, assume that $f:(X, a) \rightarrow(Y, b)$ is of effective descent and consider a chain $\gamma$ like in (WQ). Let $B_{\gamma}=\{0\} \cup\{1, \ldots, n\} \cup\{(i, 1), \ldots,(i, m)\}$ have the relations $\langle x\rangle \rightarrow x$, for every $x \in B_{\gamma}$, and $\langle 1, \ldots, n\rangle \rightarrow 0$ and $\langle(i, 1), \ldots,(i, m)\rangle \rightarrow i$. Then transitivity of $B_{\gamma}$ fails just because

$$
\langle 1, \ldots, i-1,(i, 1), \ldots,(i, m), i+1, \ldots, n\rangle \not \rightarrow 0 .
$$


The map $p_{\gamma}: B_{\gamma} \rightarrow(Y, b)$, with $p_{\gamma}(j)=y_{j}$ for $j=1, \ldots, n$, and $p_{\gamma}(i, j)=y_{j}^{i}$, for $j=1, \ldots, m$, is a monotone map, hence a morphism in PsRelAlg(W). If we form its pullback along $f$

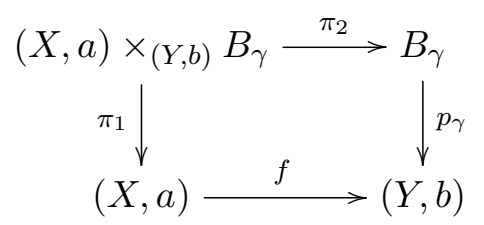

then $(X, a) \times_{(Y, b)} B_{\gamma}$ is not a multi-ordered set, by Lemma 1.2. The lack of transitivity must use an argument as in $B_{\gamma}$; hence there must exist $x_{j} \in f^{-1}\left(y_{j}\right)$, for $j=1, \ldots, n, x_{j}^{i} \in f^{-1}\left(y_{j}^{i}\right)$, for $j=1, \ldots, m$, such that $\left\langle x_{1}^{i}, \ldots, x_{m}^{i}\right\rangle \rightarrow x_{i}$ and $\left\langle x_{1}, \ldots, x_{n}\right\rangle \rightarrow x_{0}$.

We remark that, from the construction of the $p_{\gamma}$ 's, we can conclude that, for a suitable $(Y, b)$, the morphism $p: \coprod_{\gamma} 3_{\gamma} \rightarrow(Y, b)$ they induce is a weak *-quotient map, hence of effective descent, which is not a $*$-quotient map.

\section{The topology of a Relational algebra}

Based on results from [21], in this section we will recall how coproducts of relational algebras are built, concluding that both categories $\operatorname{RelAlg}(\mathbb{T})$ and PsRelAlg $(\mathbb{T})$ are (infinitely) extensive. The essential ingredient is the notion of open morphism of (pseudo-)relational algebras, which at the end of the section will guide us to the construction of a natural topology for a relational algebra.

A morphism $f:(X, a) \rightarrow(Y, b)$ is said to be open if $b^{\circ} \cdot f=T f \cdot a^{\circ}$; that is, for all $\mathfrak{y} \in T Y$ and $x \in X$,

$$
\mathfrak{y} \rightarrow f(x) \Rightarrow \exists \mathfrak{x} \in(T f)^{-1}(\mathfrak{y}): \mathfrak{x} \rightarrow x .
$$

As shown in [6], open morphisms are pullback-stable. If $(Y, b)$ is a relational algebra and $X \subseteq Y$ has the initial structure for the inclusion $f: X \rightarrow Y$, then $f$ is an open embedding if, and only if, for all $\mathfrak{y} \in T Y$, if $\mathfrak{y} \rightarrow x \in X$, then $\mathfrak{y} \in T X$ (the abuse of notation is due to the fact that, since $T$ preserves monomorphisms, $T f$ can be seen as an inclusion: see the discussion after 1.10 in $[22])$.

The following results are straightforward.

Lemma 2.1. Let $(X, a) \stackrel{f}{\longrightarrow}(Y, b) \stackrel{g}{\longrightarrow}(Z, c)$ be morphisms in $\operatorname{RelAlg}(\mathbb{T})$.

(1) If $f$ and $g$ are open, then $g \cdot f$ is open.

(2) If $g \cdot f$ is open and $f$ is surjective, then $g$ is open.

Corollary 2.2. If $f:(X, a) \rightarrow(Y, b)$ is an open morphism in $\operatorname{RelAlg}(\mathbb{T})$, then, for each open embedding $(U, \tilde{a}) \hookrightarrow(X, a)$ with $U \subseteq X$, the image of $U$ under $f$ is again an open embedding; that is, if we consider $f(U) \subseteq Y$ with the initial structure $\tilde{b}$ with respect to the inclusion into $Y$, then $(f(U), \tilde{b}) \hookrightarrow(Y, b)$ is an open embedding.

In $[21]$ it is shown that:

Theorem 2.3. Let $(X, a)$ and $\left(X_{i}, a_{i}\right)_{i \in I}$ be relational $\mathbb{T}$-algebras with $\left(\iota_{i}: X_{i} \rightarrow X\right)_{i \in I} a$ coproduct in Set. The following assertions are equivalent:

(i) $\left(\iota_{i}:\left(X_{i}, a_{i}\right) \rightarrow(X, a)\right)_{i \in I}$ is a coproduct in $\operatorname{RelAlg}(\mathbb{T})$;

(ii) for each $i \in I, \iota_{i}:\left(X_{i}, a_{i}\right) \rightarrow(X, a)$ is an open embedding. 
(The correspondent result for PsRelAlg $(\mathbb{T})$ is also valid and can be proved analogously.) From this theorem it follows easily that:

Corollary 2.4. The categories $\operatorname{RelAlg}(\mathbb{T})$ and $\operatorname{PsRelAlg}(\mathbb{T})$ are infinitely extensive.

In the sequel we will also make use of the following results, that are shown in [21]:

Proposition 2.5. (1) If $\left(f_{i}:\left(X_{i}, a_{i}\right) \rightarrow(X, a)\right)_{i \in I}$ is a set-indexed family of open morphisms in $\operatorname{RelAlg}(\mathbb{T})$, then the induced morphism $f: \coprod_{i \in I}\left(X_{i}, a_{i}\right) \rightarrow(X, a)$ is open.

(2) If $\left(f_{i}:\left(X_{i}, a_{i}\right) \rightarrow\left(Y_{i}, b_{i}\right)\right)_{i \in I}$ is a set-indexed family of morphisms in RelAlg $(\mathbb{T})$, then the morphism $\amalg f_{i}: \amalg\left(X_{i}, a_{i}\right) \rightarrow \coprod\left(Y_{i}, b_{i}\right)$ is open if and only if each $f_{i}$ is open.

Each relational $\mathbb{T}$-algebra $(X, a)$ defines a topology $\tau_{a}$ on $X$ by

$$
\tau_{a}=\{U \subseteq X \mid \text { the embedding } u:(U, \tilde{a}) \hookrightarrow(X, a) \text { is open }\} .
$$

Indeed,

$-1_{X}$ is open, and the intersection (i.e., pullback) of two open embeddings is open,

- if $\left(U_{i}\right)_{i \in I}$ is a family of elements of $\tau_{a}$, their join is formed as the image of the open morphism $\coprod U_{i} \rightarrow X$

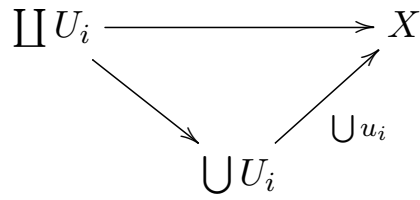

hence it is open.

Moreover, every morphism $f:(X, a) \rightarrow(Y, b)$ is a continuous map $f:\left(X, \tau_{a}\right) \rightarrow\left(Y, \tau_{b}\right)$ since open embeddings are stable under pullback. Therefore, these assignments define a functor

$$
\mathcal{O}: \operatorname{RelAlg}(\mathbb{T}) \rightarrow \operatorname{Top},(X, a) \mapsto\left(X, \tau_{a}\right), f \mapsto f .
$$

In order to construct a right adjoint to $\mathcal{O}$ we need the following auxiliary result.

Lemma 2.6. Given a relation $r: X \rightarrow Y, \mathfrak{x} \in T X$ and $\mathfrak{y} \in T Y$,

$$
\mathfrak{x}(\bar{T} r) \mathfrak{y} \Rightarrow(\forall N \subseteq Y)\left(\mathfrak{y} \in T N \Rightarrow \mathfrak{x} \in T\left(r^{\circ}(N)\right)\right),
$$

where $r^{\circ}(N)=\{x \in X \mid \exists y \in N: x r y\}$.

Theorem 2.7. The functor $\mathcal{O}$ has a right adjoint $\mathcal{C}: \operatorname{Top} \rightarrow \operatorname{RelAlg}(\mathbb{T})$, which assigns to each topological space $(X, \tau)$ the relational $\mathbb{T}$-algebra $\left(X, a_{\tau}\right)$ defined by

$$
\mathfrak{x} \rightarrow x: \Leftrightarrow \forall U \in \tau(x \in U \Rightarrow \mathfrak{x} \in T U),
$$

for any $x \in X$ and $\mathfrak{x} \in T X$.

Proof. For any $M \subseteq X, x \in M$ implies $\eta_{X}(x) \in T M$, hence $a_{\tau}$ is clearly reflexive. To show that $a_{\tau}$ is transitive, let $\mathfrak{X} \rightarrow \mathfrak{x} \rightarrow x$ in $X$, and let $x \in U \in \tau$, with $u: U \rightarrow X$ the inclusion map. Then $\mathfrak{x} \in T U$, hence $\mathfrak{X} \in T\left(a_{\tau}^{\circ}(U)\right)$, by the lemma above, and then $\mathfrak{X} \in T T U$ since $a_{\tau}^{\circ}(U) \subseteq T U$ by definition of $a_{\tau}$. Therefore the commutativity of the diagram

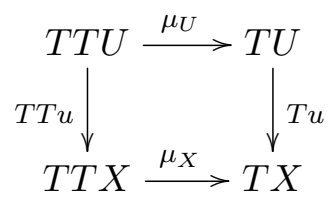

gives $\mu_{X}(\mathfrak{X}) \in T U$, hence $\mu_{X}(\mathfrak{X}) \rightarrow x$ as claimed. 
Now, let $f:(X, \tau) \rightarrow(Y, \sigma)$ be a continuous map, and $\mathfrak{x} \rightarrow x$ in $\left(X, a_{\tau}\right)$. For any $V \in \sigma$ with $f(x) \in V, f^{-1}(V)$ is open in $X$ and $x \in f^{-1}(V)$, hence $\mathfrak{x} \in T\left(f^{-1}(V)\right)$, and then $T f(\mathfrak{x}) \in T V$. Therefore $f:\left(X, a_{\tau}\right) \rightarrow\left(Y, a_{\sigma}\right)$ is a morphism in $\operatorname{RelAlg}(\mathbb{T})$.

For a relational algebra $(X, a)$, the identity map $(X, a) \rightarrow \mathcal{C O}(X, a)$ is a morphism in $\operatorname{RelAlg}(\mathbb{T})$, since, whenever $\mathfrak{x} \rightarrow x$ in $(X, a)$ and $U \in \tau_{a}$ with $x \in U$, then $\mathfrak{x} \in T U$ by definition of $\tau_{a}$. Therefore $\mathfrak{x} \rightarrow x$ in $\mathcal{C O}(X, a)$.

Likewise, for a topological space $(X, \tau)$, the identity map $\mathcal{O} \mathcal{C}(X, \tau) \rightarrow(X, \tau)$ is continuous: if $U \in \tau$ and $\mathfrak{x} \rightarrow x$ in $\left(X, a_{\tau}\right)$, necessarily $\mathfrak{x} \in T U$, which means exactly $U$ is open in $\mathcal{O} \mathfrak{C}(X, \tau)$.

Proposition 2.8. If $f:(X, a) \rightarrow(Y, b)$ is an open morphism in $\operatorname{RelAlg}(\mathbb{T})$, then $\mathcal{O}(f)$ is open. If $f$ is an embedding, then also the reverse implication holds.

Proof. Follows from Corollary 2.2.

Remark 2.9. An embedding $m: M \rightarrow X$ in $\operatorname{RelAlg}(\mathbb{T})$ will be called an $\mathcal{O}$-embedding if $\mathcal{O}(m): \mathcal{O}(M) \rightarrow \mathcal{O}(X)$ is a topological embedding. We observe that open embeddings and split monomorphisms are $\mathcal{O}$-embeddings.

For a morphism $f:(X, a) \rightarrow(Y, b)$, if in condition $(\mathrm{C})$ uniqueness of $\mathfrak{x}$ is assumed, that is, if, for all $\mathfrak{y} \in T Y$ and $x \in X$,

$$
\mathfrak{y} \rightarrow f(x) \Rightarrow \exists ! \mathfrak{x} \in(T f)^{-1}(\mathfrak{y}): \mathfrak{x} \rightarrow x,
$$

then $f$ is said to be a discrete fibration. In case $T$ is cartesian, it is easy to check that discrete fibrations are pullback stable, but this is not true for a general monad, not even for the ultrafilter monad (cf. [8,9]). Pullback-stable discrete fibrations are called étale morphisms in [9]. Although this notion recovers the notion of étale continuous map - or local homeomorphism - when $\mathbb{T}$ is the ultrafilter monad, in general the functor $\mathcal{O}$ does not preserve étale morphisms (see Example 2.15). Here we will consider the stronger notion of local isomorphism. By local isomorphism we mean a morphism $f:(X, a) \rightarrow(Y, b)$ such that, for each $x \in X$, there exists an open embedding $u: U \rightarrow X$ with $x \in U$ and $f \cdot u$ an open embedding.

Proposition 2.10. If $(X, a)$ and $(Y, b)$ are relational $\mathbb{T}$-algebras, a morphism $f:(X, a) \rightarrow(Y, b)$ is a local isomorphism if and only if $\mathcal{O}(f)$ is.

Making use of the functor $\mathcal{O}$, we can conclude immediately:

Corollary 2.11. In $\operatorname{RelAlg}(\mathbb{T})$ :

(1) Every open embedding is a local isomorphism; in particular, every coproduct injection is a local isomorphism.

(2) If $\left(f_{i}: X_{i} \rightarrow Y\right)_{i \in I}$ is a family of local isomorphisms, then the induced morphism $f: \amalg X_{i} \rightarrow$ $Y$ in the coproduct is a local isomorphism.

(3) If $\left(f_{i}: X_{i} \rightarrow Y_{i}\right)_{i \in I}$ is a family of local isomorphisms, then $\amalg f_{i}: \amalg X_{i} \rightarrow \amalg Y_{i}$ is a local isomorphism.

Lemma 2.12. In $\operatorname{RelAlg}(\mathbb{T})$ local isomorphisms are pullback stable.

Proof. Given a pullback diagram (B) with $f$ a local isomorphism, let $(x, z) \in X \times_{Y} Z$, and let $u: U \rightarrow X$ be an open embedding such that $f \cdot u$ is an open embedding. Then, if $u^{\prime}: U^{\prime} \rightarrow X \times_{Y} Z$ is the pullback of $u$ along $\pi_{1}$, then $u^{\prime}$, as well as $\pi_{2} \cdot u^{\prime}$, are open embeddings.

Lemma 2.13. In $\operatorname{RelAlg}(\mathbb{T})$ every local isomorphism is étale; in particular, it is open. 
Proof. By pullback stability of local isomorphisms, it is enough to show that every local isomorphism $f:(X, a) \rightarrow(Y, b)$ is a discrete fibration. Let $\mathfrak{y} \rightarrow f(x)$, with $\mathfrak{y} \in T Y$ and $x \in X$. Let $u: U \rightarrow X$ be an open embedding with $x \in U$ and $f \cdot u$ an open embedding. Then $\mathfrak{y}=T(f \cdot u)(\mathfrak{u})$ for a unique $\mathfrak{u} \in T U$ with $\mathfrak{u} \rightarrow x$, hence $T u(\mathfrak{u}) \in T X$ is unique such that $T u(\mathfrak{u}) \rightarrow x$ and $T f(T u(\mathfrak{u}))=\mathfrak{y}$.

Lemma 2.14. Given a commutative diagram

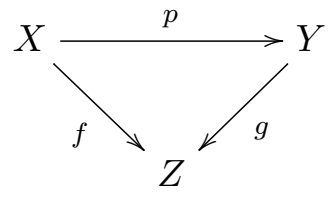

in $\operatorname{RelAlg}(\mathbb{T})$ :

(1) if $f$ and $g$ are local isomorphisms, then $p$ is a local isomorphism;

(2) if $f$ is a local isomorphism and $p$ is an open surjection, then $g$ is a local isomorphism.

Proof. It follows from Lemma 6.4.5 of [2], making use of the functor $\mathcal{O}$.

Example 2.15. In $M$-Ord an equivariant map $f:(X, a) \rightarrow(Y, b)$ is open (étale) provided that:

$$
\forall x_{0} \in X \forall y_{1} \in Y \forall m \in M\left(\left(m, y_{1}\right) \rightarrow f\left(x_{0}\right) \Rightarrow \exists(!) x_{1} \in f^{-1}\left(y_{1}\right):\left(m, x_{1}\right) \rightarrow x_{0}\right)
$$

It is a local isomorphism if

$$
\forall x_{0} \in X \forall y_{1} \in Y\left(\left(\exists m \in M:\left(m, y_{1}\right) \rightarrow f\left(x_{0}\right)\right) \Rightarrow\left(f^{-1}\left(y_{1}\right)=\left\{x_{1}\right\} \text { and }\left(m, x_{1}\right) \rightarrow x_{0}\right)\right)
$$

If $M$ is non-trivial there are étale maps which are not local isomorphisms. For instance, for $m, n \in M$ with $m \neq n$, consider $X=(\{0,1,2\}, a)$ with non-trivial relations $(m, 1) \rightarrow 0$ and $(n, 2) \rightarrow 0$, and $Y=(\{0,1\}, b)$, with non-trivial relations $(m, 1) \rightarrow 0$ and $(n, 1) \rightarrow 0$; the equivariant map $f:(X, a) \rightarrow(Y, b)$ with $f(1)=f(2)=1$ and $f(0)=0$ is étale but it is not a local isomorphism. Hence, by Proposition 2.10, $\mathcal{O}(f)$ is not étale, which shows that $\mathcal{O}$ does not preserve étale morphisms.

\section{Connected Relational algebras}

For the sake of completeness, we start this section by proving a result that can be found in $[17]$.

Theorem 3.1. Let $\mathbf{C}$ be a finitely complete and infinitely extensive category. For an object $A$ of $\mathbf{C}$, the following conditions are equivalent:

(i) $\operatorname{hom}(A,-)$ preserves coproducts;

(ii) for any morphism $f: A \rightarrow \coprod_{i \in I} B_{i}$, there exist $j \in I$ and $t: A \rightarrow B_{j}$ such that $f=\iota_{j} \cdot t$, with $\iota_{j}: B_{j} \rightarrow \coprod_{i} B_{i}$ the coprojection;

(iii) $A \nsubseteq 0$ and $A \cong B+C \Rightarrow A \cong B$ or $A \cong C$, that is the isomorphism $A \rightarrow B+C$ factors through one of the coprojections;

(iv) $A \neq 0$ and $A \cong B+C \Rightarrow B \cong 0$ or $C \cong 0$.

Proof. Clearly (i) $\Rightarrow$ (ii) $\Rightarrow$ (iii), and (iii) $\Leftrightarrow$ (iv) since coproducts are disjoint. 
(iv) $\Rightarrow$ (i): Let $f: A \rightarrow \coprod_{i} B_{i}$ be a morphism in C. If we consider, for every $j \in I$, the pullback diagram

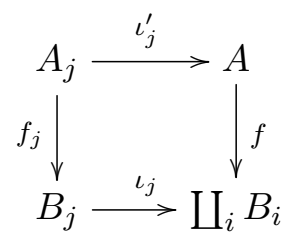

we know that $A \cong \coprod_{i} A_{i}$; hence all but one are initial objects, and so $f$ factors through the only non-trivial coprojection.

Definitions 3.2. In a finitely complete and infinitely extensive category $\mathbf{C}$, an object $A$ satisfying the (equivalent) conditions above is said to be connected. An extremal monomorphism $C \rightarrow X$ is a connected component of $X$ if it has connected domain and it does not factor properly through an extremal monomorphism with connected domain.

We remark that, when $\mathbf{C}=$ Top, connected space and connected component take their usual topological meaning.

Proposition 3.3. For a relational $\mathbb{T}$-algebra $(X, a)$, the following conditions are equivalent:

(i) $(X, a)$ is connected;

(ii) $\mathcal{O}(X)$ is a connected space.

Proof. (i) $\Rightarrow$ (ii): Let $(X, a)$ be connected, with $X \neq \emptyset$. If $\mathcal{O}(X)$ is the disjoint union of two open subspaces $U, V$, then, since $\mathcal{O}$ preserves coproducts,

$$
\mathcal{O}(X)=\mathcal{O}(U)+\mathcal{O}(V) \cong \mathcal{O}(U+V),
$$

where $U$ and $V$ have the relational structures induced by $a$. Hence, the morphism $U+V \rightarrow X$ is an open bijection, and so an isomorphism. Therefore $U=\emptyset$ or $V=\emptyset$, and we conclude that $\mathcal{O}(X)$ is a connected space.

(ii) $\Rightarrow$ (i): If $\mathcal{O}(X)$ is connected, with $X \neq \emptyset$, and $f:(X, a) \rightarrow(B+C, b)$ is an isomorphism, then $\mathcal{O}(f): \mathcal{O}(X) \rightarrow \mathcal{O}(B+C) \cong \mathcal{O}(B)+\mathcal{O}(C)$ is an isomorphism, and so $\mathcal{O}(B)=\emptyset$ or $\mathcal{O}(C)=\emptyset$, that is $B=\emptyset$ or $C=\emptyset$.

Corollary 3.4. If $T 1 \cong 1$, then the product of two connected relational algebras is connected.

Proof. If $X$ and $Y$ are connected relational algebras, for each $x_{0} \in X$ and $y_{0} \in Y$, both $X \cong$ $X \times 1 \rightarrow X \times Y$ and $Y \cong 1 \times Y \rightarrow X \times Y$, mapping $(x, *)$ to $\left(x, y_{0}\right)$ and $(*, y)$ to $\left(x_{0}, y\right)$, respectively, are split monomorphisms, hence $\mathcal{O}$-embeddings. (Here 1 is the terminal object $(\{*\}, \top)$ of $\operatorname{RelAlg}(\mathbb{T})$, that is $\top(\mathfrak{x}, *)=\top$ for every $\mathfrak{x} \in T(\{*\})$.) Therefore their images under $\mathcal{O}, \mathcal{O}(X) \rightarrow \mathcal{O}(X \times Y)$ and $\mathcal{O}(Y) \rightarrow \mathcal{O}(X \times Y)$, define connected subspaces of $\mathcal{O}(X \times Y)$. Hence $\mathcal{O}(X \times Y)$ can be written as a union of connected subspaces with non-empty intersection and so it is connected. Finally, by the proposition above, $X \times Y$ is connected.

We point out that the condition $T 1 \cong 1$ is essential here. In fact, if we consider, for instance, the word monad $\mathbb{W}$ and the relational $\mathbb{W}$-algebra $E=(\{*\}, e)$ with $e(\mathfrak{x}, *)=\top$ only if $\mathfrak{x}=\langle *\rangle$, then, for $X=(\{x, y\}, a)$ with $a(\langle x, y\rangle, x)=\top, X$ is connected but $X \times E$ is not.

Corollary 3.5. The following assertions are equivalent, for a relational $\mathbb{T}$-algebra:

(i) $(X, a)$ is a coproduct of connected relational $\mathbb{T}$-algebras;

(ii) $(X, a)$ is the coproduct of its connected components;

(iii) $\mathcal{O}(X)$ is the coproduct of its connected components. 
Recall that a topological space $X$ is an Alexandroff space if every point in $X$ has a smallest (open) neighbourhood (or, equivalently, if the intersection of open subsets of $X$ is open).

Proposition 3.6. (1) Every Alexandroff space has open connected components.

(2) If the functor $T:$ Set $\rightarrow$ Set preserves intersections, then $\mathcal{O}(X)$ is an Alexandroff space, for every relational $\mathbb{T}$-algebra $(X, a)$.

Proof. (1) If $X$ is an Alexandroff space, and $C_{x}$ is the connected component of $x \in X$, then it is open, since it is an intersection of open subsets:

$$
C_{x}=\bigcap_{y \in X \backslash C_{x}} X \backslash C_{y} .
$$

(2) Let $U_{i} \subseteq X, i \in I$, be open in $\mathcal{O}(X)$, and $U=\bigcap_{i \in I} U_{i}$. Let $\mathfrak{x} \in T X$ with $\mathfrak{x} \rightarrow x \in U$. Since $x \in U_{i}$ for every $i \in I, \mathfrak{x} \in T U_{i}$ because $U_{i} \hookrightarrow X$ is open. Therefore $\mathfrak{x} \in \bigcap_{i \in I} T U_{i}=T\left(\bigcap_{i \in I} U_{i}\right)=$ $T U$.

Corollary 3.7. If $T$ preserves intersections, then every relational $\mathbb{T}$-algebra is the coproduct of its connected components.

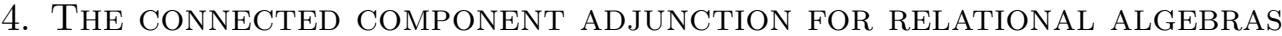

Let $\operatorname{Conn}(\mathbb{T})$ be the subcategory of connected objects of $\operatorname{RelAlg}(\mathbb{T})$. In this section we use the category $\operatorname{Fam}(\operatorname{Conn}(\mathbb{T}))$, which we will often think of as a full subcategory of $\operatorname{RelAlg}(\mathbb{T})$.

For any monad $\mathbb{T}$, we will study the semi-left exact adjunction

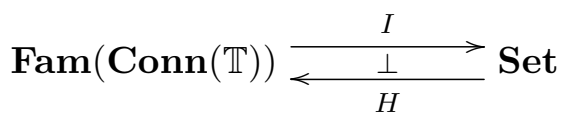

with $I\left(\left(X_{j}, a_{j}\right)_{j \in J}\right)=J$ and $H(X)=\coprod_{X} 1$, where 1 is the terminal object, as described in [2, Section 6.1] and [18].

Proposition 4.1. If $T 1 \cong 1$, then the adjunction $I \dashv H$ has stable units.

Proof. Given a diagram

$$
\begin{gathered}
\coprod_{k \in K} D_{k} \\
\downarrow^{g} \\
X=\coprod_{j \in J} C_{j} \stackrel{\eta_{X}}{\longrightarrow} \coprod_{j \in J} 1=H I(X)
\end{gathered}
$$

$g$ is just a map $K \rightarrow J$, since $D_{k}$ is connected. Hence, its pullback in $\operatorname{RelAlg}(\mathbb{T})$ is the coproduct of the pullbacks of pairs $C_{j} \longrightarrow 1 \longleftarrow D_{k}$ :

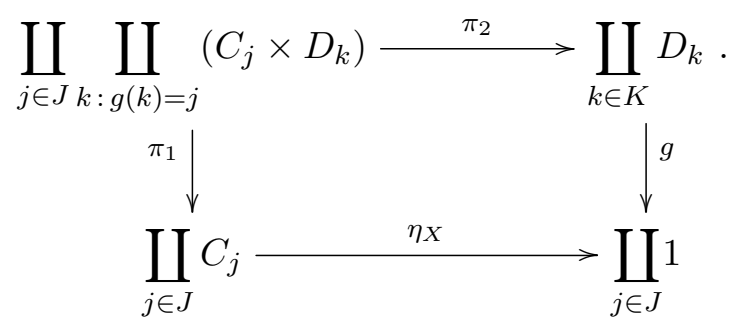

Here $\pi_{2}=\left(\pi_{2}^{k}\right)_{k \in K}: \coprod_{k \in K}\left(C_{g(k)} \times D_{k}\right) \rightarrow \coprod_{k \in K} D_{k}$. Since $C_{g(k)} \times D_{k}$ is connected by Corollary 3.4, this is a pullback in $\operatorname{Fam}(\operatorname{Conn}(\mathbb{T}))$ and, moreover, $I\left(\pi_{2}\right)=(1: K \rightarrow K)$. 
By Corollary 3.7, $\operatorname{RelAlg}(\mathbb{T})$ is isomorphic to the category $\operatorname{Fam}(\operatorname{Conn}(\mathbb{T}))$, whenever the functor $T$ preserves intersections. This is the case of the monads $\mathbb{I}, \mathbb{M}$ and $\mathbb{W}$, that will be further studied in the next section. The monad $\mathbb{U}$ does not preserve intersections and the corresponding study of covering morphisms requires some additional assumptions, as explored in Section 6.

\section{COVERING MORPHiSMS}

Throughout this section we assume that the functor $T$ is cartesian and preserves intersections. By Corollary 3.7, $\operatorname{RelAlg}(\mathbb{T})$ is isomorphic to $\operatorname{Fam}(\operatorname{Conn}(\mathbb{T}))$, so that the connected component adjunction is defined in all the category of relational algebras

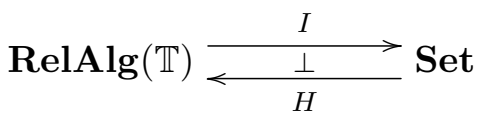

We recall that a morphism $f:(X, a) \rightarrow(Y, b)$ is said to be a trivial covering if the following diagram

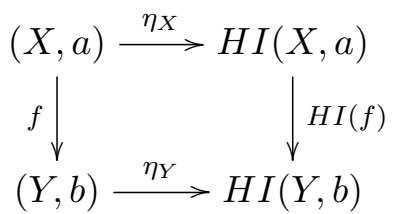

is a pullback. (This implies, in particular, that the (co)restrictions of $f$ to connected components are isomorphisms.) It is said to be a covering if there is an effective descent morphism $p:(Z, c) \rightarrow$ $(Y, b)$ such that the pullback of $f$ along $p$ is a trivial covering. (For details see [2, Section 6.5].)

Proposition 5.1. Let $T$ be cartesian and preserve intersections.

(1) If $f:(X, a) \rightarrow(Y, b)$ is a trivial covering, then:

(a) it is a local isomorphism;

(b) it has closed image, in the sense that, for any $\mathfrak{x} \in T X \backslash T \emptyset$ and $y \in Y$, if $T f(\mathfrak{x}) \rightarrow y$ then $y \in f(X)$;

(c) it has separated fibres, in the sense that, for any $\mathfrak{x} \in T X \backslash T \emptyset$, if $\mathfrak{x} \rightarrow x$ and $\mathfrak{x} \rightarrow x^{\prime}$ with $f(x)=f\left(x^{\prime}\right)$, then $x=x^{\prime}$.

(2) If, moreover, $T$ preserves coproducts, then every trivial covering is a discrete opfibration.

Proof. (1a) First we show that, for any map $g: Z \rightarrow W$ in Set, $H(g): \coprod_{Z} 1 \rightarrow \coprod_{W} 1$ is a local isomorphism. Indeed, given $z \in Z, 1_{z} \stackrel{\iota_{z}}{\longrightarrow} \coprod_{Z} 1$ is open and $H(g) \cdot \iota_{z}=\iota_{g(z)}: 1_{g(z)} \rightarrow \coprod_{W} 1$ is an open embedding too. Now the conclusion follows from pullback stability of local isomorphisms.

(1b) Again we start by proving that $H(g)$ has closed image, for any map $g: Z \rightarrow W$. Let $\mathfrak{Z} \in T\left(\coprod_{Z} 1\right) \backslash T \emptyset$ be such that $T(H(g))(\mathfrak{Z}) \rightarrow * w$, for some $w \in W$. Then $\mathfrak{Z} \in T\left(\coprod_{g^{-1}(w)} 1\right) \backslash T \emptyset$, since the following diagram

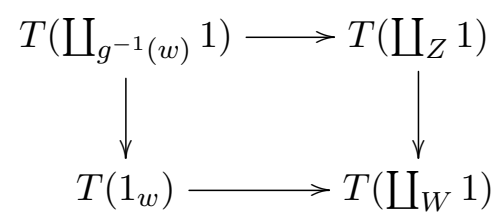

is a pullback. Therefore $g^{-1}(w) \neq \emptyset$ and the result follows. Next, to complete the proof, we show that this property is stable under pullback. Given a pullback diagram (B) and $\mathfrak{w} \in$ $T\left(X \times_{Y} Z\right) \backslash T \emptyset$ such that $T \pi_{1}(\mathfrak{w}) \rightarrow x$ in $X$, if $g$ has closed image then, since $T \pi_{2}(\mathfrak{w}) \in T Z \backslash T \emptyset$ and $T f\left(T \pi_{2}(\mathfrak{w})\right) \rightarrow f(x)$, there exists $z \in Z$ with $g(z)=f(x)$. Then $(x, z) \in X \times_{Y} Z$ and $\pi_{1}(x, z)=x$. 
(1c) $H(g)$ has separated fibres: assume $\mathfrak{Z} \rightarrow *_{z}$ and $\mathfrak{Z} \rightarrow *_{z^{\prime}}$, with $\mathfrak{Z} \notin T \emptyset$ and $z \neq z^{\prime}$; then $\mathfrak{Z} \in T\left(1_{z}\right) \cap T\left(1_{z^{\prime}}\right)=T \emptyset$. Moreover, this property is stable under pullback: given a pullback diagram (B) and $\mathfrak{w} \in T\left(X \times_{Y} Z\right) \backslash T \emptyset$ such that $\mathfrak{w} \rightarrow(x, z)$ and $\mathfrak{w} \rightarrow\left(x, z^{\prime}\right)$, then $T \pi_{2}(\mathfrak{w}) \notin T \emptyset$, $T \pi_{2}(\mathfrak{w}) \rightarrow z$ and $T \pi_{2}(\mathfrak{w}) \rightarrow z^{\prime}$, therefore $z=z^{\prime}$.

(2) It is enough to show that $H(g)$, for any map $g: Z \rightarrow W$, is a discrete opfibration, since this property is stable under pullback. Let $\mathfrak{Z} \in T\left(\coprod_{Z} 1_{z}\right)=\coprod_{Z} T\left(1_{z}\right)$ be such that $T(H(g))(\mathfrak{Z}) \rightarrow *_{w}$ for some $w \in W$. Since $\mathfrak{Z} \in T\left(1_{z}\right)$ for a unique $z \in Z$, we have $\mathfrak{Z} \rightarrow *_{z}$ and necessarily $H(g)\left(*_{z}\right)=*_{w}$.

Proposition 5.2. Given a pullback diagram (B) with $f$ a final morphism:

(1) If $\pi_{1}$ is a discrete (op)fibration, then $g$ is a discrete (op)fibration.

(2) If $\pi_{1}$ has closed image, then $g$ has closed image.

(3) If $\pi_{1}$ has separated fibres, then $g$ has separated fibres.

Proof. (1) Let $\mathfrak{y} \in T Y$ and $z \in Z$ with $\mathfrak{y} \rightarrow g(z)$. Finality of $f$ gives $\mathfrak{x} \in T X$ and $x \in X$ with $\mathfrak{x} \rightarrow x, T f(\mathfrak{x})=\mathfrak{y}$ and $f(x)=g(z)$. Then $\mathfrak{x} \rightarrow \pi_{1}(x, z)$, and so there exists a unique $\mathfrak{w} \in T\left(X \times_{Y} Z\right)$ such that $\mathfrak{w} \rightarrow(x, z)$ and $T \pi_{1}(\mathfrak{w})=\mathfrak{x} ;$ hence $\mathfrak{Z}:=T \pi_{2}(\mathfrak{w}) \rightarrow z$ and $T g(\mathfrak{Z})=\mathfrak{y}$. To show uniqueness of such $\mathfrak{Z}$ we take any $\mathfrak{Z}^{\prime}$ with $\mathfrak{Z}^{\prime} \rightarrow z$ and $T g\left(\mathfrak{Z}^{\prime}\right)=\mathfrak{y}$. Since $T f(\mathfrak{x})=T g\left(\mathfrak{Z}^{\prime}\right)$, by (BC) there exists $\mathfrak{w}^{\prime} \in T\left(X \times_{Y} Z\right)$ such that $T \pi_{1}\left(\mathfrak{w}^{\prime}\right)=\mathfrak{x}$ and $T \pi_{2}\left(\mathfrak{w}^{\prime}\right)=\mathfrak{Z}^{\prime}$. By definition of the pullback structure, $\mathfrak{w}^{\prime} \rightarrow(x, z)$. Therefore $\mathfrak{w}^{\prime}$ must be equal to $\mathfrak{w}$, and so $\mathfrak{Z}^{\prime}=\mathfrak{Z}$ as claimed.

For discrete opfibrations the proof is similar.

(2) Let $\mathfrak{Z} \in T Z \backslash T \emptyset$ and $y \in Y$ with $T g(\mathfrak{Z}) \rightarrow y$ in $Y$. Again, since $f$ is final, there exist $\mathfrak{x} \in T X, x \in X$ with $\mathfrak{x} \rightarrow x, T f(\mathfrak{x})=T g(\mathfrak{Z})$ and $f(x)=y$. By $(\mathrm{BC}), \mathfrak{x}=T \pi_{1}(\mathfrak{w})$ and $\mathfrak{Z}=T \pi_{2}(\mathfrak{w})$ for some $\mathfrak{w} \in T\left(X \times_{Y} Z\right) \backslash T \emptyset$. Then $x=\pi_{1}(x, z)$ for some $z \in Z$ because $\pi_{1}$ has closed image, and therefore $y=g(z)$.

(3) Let $\mathfrak{Z} \in T Z \backslash T \emptyset$, with $\mathfrak{Z} \rightarrow z, \mathfrak{Z} \rightarrow z^{\prime}$ and $g(z)=g\left(z^{\prime}\right)$. Since $f$ is final, there exist $\mathfrak{x} \rightarrow x$ with $T f(\mathfrak{x})=T g(\mathfrak{Z})$ and $f(x)=g(z)$. Therefore $\mathfrak{w}=(\mathfrak{x}, \mathfrak{Z}) \notin T \emptyset, \mathfrak{w} \rightarrow(x, z)$ and $\mathfrak{w} \rightarrow\left(x, z^{\prime}\right)$, which implies $(x, z)=\left(x, z^{\prime}\right)$.

Using Theorem 1.3, we conclude the following.

Corollary 5.3. Let $T$ be cartesian and preserve intersections, and let $\eta$ have (BC) for relations with finite fibres.

(1) Every covering is a discrete fibration with closed image and separated fibres.

(2) If, moreover, $T$ preserves coproducts, then every covering is a discrete fibration and a discrete opfibration.

Next we characterize coverings for the connected component adjunction in case $T$ is the multiplication by a monoid or the free monoid monad.

\section{Examples 5.4.}

I. $M$-ordered sets. For a given monoid $M, \mathbb{M}$ is cartesian, preserves intersections and coproducts.

Theorem 5.5. An equivariant map $f:(X, a) \rightarrow(Y, b)$ is a covering in $M$-Ord if and only if it is a discrete fibration and a discrete opfibration.

Proof. Since $\mathbb{M}$ is cartesian, preserves intersections and coproducts, coverings are discrete fibrations and discrete opfibrations. To show the converse, let $f:(X, a) \rightarrow(Y, b)$ be a discrete fibration and discrete opfibration. For every chain $\gamma$, given by $\left(m, n, y_{2}\right) \rightarrow\left(m, y_{1}\right) \rightarrow y_{0}$ in 
$Y$, let $3_{\gamma}$ be as in the proof of Theorem 1.8, and consider the inclusion $p_{\gamma}: 3_{\gamma} \rightarrow(Y, b)$, with $p_{\gamma}(i)=y_{i}$. If one forms the coproduct $\coprod_{\gamma} 3_{\gamma}$, where $\gamma$ is any chain as before, the equivariant map $p: \coprod_{\gamma} 3_{\gamma} \rightarrow(Y, b)$ induced by $\left(p_{\gamma}\right)$ is a $*$-quotient map by construction, hence an effective descent morphism. Moreover, the pullback $\pi_{1}$ of $f$ along $p$

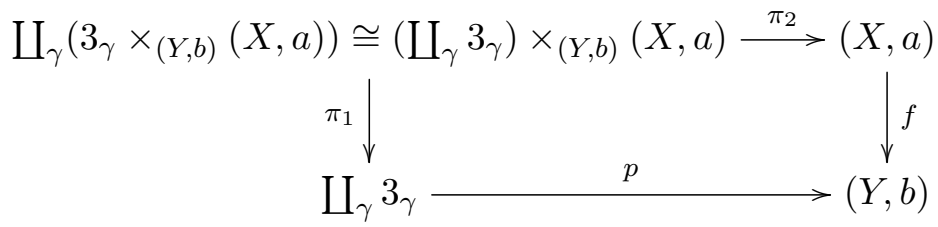

is a trivial covering. To show this we study, for a given chain $\gamma$, the corresponding $M$-ordered set $3_{\gamma} \times_{(Y, b)}(X, a)$. Since $f$ is both a discrete fibration and a discrete opfibration, if $3_{\gamma} \times_{(Y, b)}(X, a)$ is non-empty, there exist unique $x_{2} \in f^{-1}\left(y_{2}\right), x_{1} \in f^{-1}\left(y_{1}\right)$ and $x_{0} \in f^{-1}\left(y_{0}\right)$ such that $\left(m, n, x_{2}\right) \rightarrow\left(m, x_{1}\right) \rightarrow x_{0}$. That is, $3_{\gamma} \times_{(Y, b)}(X, a)$ is isomorphic to $3_{\gamma}$, and the restriction of $\pi_{1}$ to $3_{\gamma} \times_{(Y, b)}(X, a)$ gives this isomorphism. This means exactly that $\pi_{1}$ is a trivial covering, as claimed.

We remark that, when $M=\{e\}$, this theorem gives the already known characterization of covering maps for the adjunction defined in Ord (cf. [24]).

II. Multi-ordered sets. The monad $\mathbb{W}$ is cartesian and preserves intersections, but it does not preserve coproducts.

Theorem 5.6. A monotone map $f:(X, a) \rightarrow(Y, b)$ is a covering in MultiOrd if and only if it is a discrete fibration verifying the following conditions:

(SC) for each $y_{0}, \ldots, y_{n} \in Y$, if $\left\langle y_{1}, \ldots, y_{n}\right\rangle \rightarrow y_{0}$ and $f(X) \cap\left\{y_{0}, y_{1}, \ldots, y_{n}\right\} \neq \emptyset$, then $\left\{y_{0}, \ldots, y_{n}\right\} \subseteq f(X)$

(SF) for each $x_{0}, x_{1}, \ldots, x_{n}, x_{0}^{\prime}, x_{1}^{\prime}, \ldots, x_{n}^{\prime}$ with $f\left(x_{j}\right)=f\left(x_{j}^{\prime}\right)$, for all $j$, and $x_{j}=x_{j}^{\prime}$, for some $j$, if $\left\langle x_{1}, \ldots, x_{n}\right\rangle \rightarrow x_{0}$ and $\left\langle x_{1}^{\prime}, \ldots, x_{n}^{\prime}\right\rangle \rightarrow x_{0}^{\prime}$, then, for all $j, x_{j}=x_{j}^{\prime}$.

Proof. To conclude that every covering has (SC) and (SF) we observe that, for every map $g: Z \rightarrow W, H(g)$ has these properties, which are stable under pullback, and descend along final maps.

To prove the converse we will use results of 1.5.IV. Let $f$ be a discrete fibration satisfying (SC) and (SF). For each chain $\gamma$ as in (WQ), let $C_{\gamma}$ be as $B_{\gamma}$ (as in the proof of Theorem 1.10) plus the relation

$$
\langle 1, \ldots, i-1,(i, 1), \ldots,(i, m), i+1, \ldots, n\rangle \rightarrow 0,
$$

so that it is a multi-ordered set, with $p_{\gamma}: C_{\gamma} \rightarrow(Y, b)$ defined as in the proof of Theorem 1.10. Then the morphism $p: \coprod_{\gamma} C_{\gamma} \rightarrow(Y, b)$, defined by all the $p_{\gamma}$ 's, is a weak *-quotient map, by construction, hence it is of effective descent. Moreover, in the pullback of $f$ along $p$

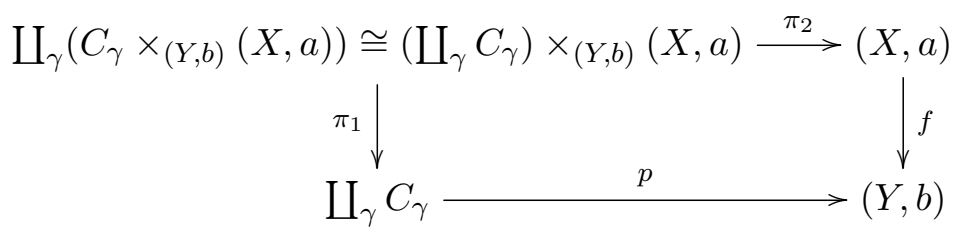

$\pi_{1}$ is a trivial covering: for each chain $\gamma$, if $C_{\gamma} \times_{(Y, b)}(X, a)$ is non-empty, then (SC) implies that the (co)restriction $\bar{\pi}_{1}: C_{\gamma} \times_{(Y, b)}(X, a) \rightarrow C_{\gamma}$ is surjective, hence final because it is a discrete fibration; to prove that $\bar{\pi}_{1}$ is injective, we observe that, if $x_{j}, x_{j}^{\prime} \in \bar{\pi}_{1}^{-1}(j)$, for $j \neq 0$, $i$, then, since the domain is connected, necessarily there exist $\left\langle x_{1}, \ldots, x_{n}\right\rangle \rightarrow x_{0},\left\langle x_{1}^{\prime}, \ldots, x_{n}^{\prime}\right\rangle \rightarrow x_{0}$, and 
then (SF) gives $x_{j}=x_{j}^{\prime}$; if $x_{0}, x_{0}^{\prime} \in \bar{\pi}_{1}^{-1}(0)$, connectedness of the domain, together with (SF), gives $x_{0}=x_{0}^{\prime}$. Finally, for $x_{i}^{j}, \bar{x}_{i}^{j} \in \bar{\pi}_{1}^{-1}(i, j)$ we repeat the argument used above.

\section{The CONNECTED COMPONENT ADJUNCTION FOR LOCALLY CONNECTED RELATIONAL}

\section{ALGEBRAS}

In general, $\operatorname{Fam}(\operatorname{Conn}(\mathbb{T}))$, as a (full) subcategory of $\operatorname{RelAlg}(\mathbb{T})$, is not closed under open embeddings. In order to consider the largest such subcategory of $\operatorname{RelAlg}(\mathbb{T})$ contained in $\operatorname{Fam}(\operatorname{Conn}(\mathbb{T}))$, we say that a relational $\mathbb{T}$-algebra $(X, a)$ is locally connected if, for every open embedding $U \rightarrow X, U$ belongs to $\operatorname{Fam}(\operatorname{Conn}(\mathbb{T}))$, that is, $U$ is a coproduct of connected relational $\mathbb{T}$-algebras.

Proposition 6.1. A relational $\mathbb{T}$-algebra $(X, a)$ is locally connected if and only if $\mathcal{O}(X)$ is a locally connected space.

Proof. This statement relies on the facts that, for every $U \subseteq X$, the embedding $u: U \rightarrow X$ is open if and only if $\mathcal{O}(u): \mathcal{O}(U) \rightarrow \mathcal{O}(X)$ is an open embedding, and that $U \in \operatorname{Fam}(\operatorname{Conn}(\mathbb{T}))$ if and only if $\mathcal{O}(U)$ is a coproduct of connected spaces: see Remark 2.9 and Corollary 3.5.

From now on we denote by $\mathbf{L C}(\mathbb{T})$ the full subcategory of $\operatorname{RelAlg}(\mathbb{T})$ of locally connected relational $\mathbb{T}$-algebras, and by $\mathbf{C L C}(\mathbb{T})$ the full subcategory of $\operatorname{RelAlg}(\mathbb{T})$ of connected and locally connected relational $\mathbb{T}$-algebras.

Lemma 6.2. $\mathbf{L C}(\mathbb{T})$ is closed under open embeddings and coproducts in $\operatorname{RelAlg}(\mathbb{T})$.

Proof. That local connectedness is stable under open embeddings follows directly from the definition. To show that a coproduct of locally connected relational $\mathbb{T}$-algebras is locally connected we use extensivity of $\operatorname{RelAlg}(\mathbb{T})$ and pullback stability of open embeddings.

Corollary 6.3. The categories $\mathbf{L C}(\mathbb{T})$ and $\operatorname{Fam}(\mathbf{C L C}(\mathbb{T}))$ are equivalent.

Proof. Using the embedding $\operatorname{Fam}(\operatorname{Conn}(\mathbb{T})) \rightarrow \operatorname{RelAlg}(\mathbb{T})$, we want to show that $X \cong \coprod_{i \in I} C_{i}$, with $C_{i}$ connected and locally connected if, and only if, $X$ is locally connected. That $\coprod_{i \in I} C_{i}$ is locally connected follows from the stability under coproducts of locally connected relational $\mathbb{T}$-algebras. Conversely, if $X$ is locally connected, then $X \cong \coprod_{i \in I} D_{i}$, with $D_{i}$ connected for every $i \in I$; since coproduct injections are open embeddings, $D_{i}$ is also locally connected.

The category $\mathbf{L C}(\mathbb{T})$ is not closed under pullbacks in $\operatorname{RelAlg}(\mathbb{T})$. To remedy this, as done in $[2$, Section 6], from now on we restrict our study to local isomorphisms, working on the category $\mathbf{L C}_{l i}(\mathbb{T})$ of locally connected relational $\mathbb{T}$-algebras and local isomorphisms.

Similarly to Corollary 6.3, we have that:

Lemma 6.4. $\operatorname{LC}_{l i}(\mathbb{T}) \cong \operatorname{Fam}\left(\operatorname{CLC}_{l i}(\mathbb{T})\right)$.

The following result follows directly from the corresponding result in Top, using Propositions 6.1 and 2.10 .

Lemma 6.5. If $f:(X, a) \rightarrow(Y, b)$ is a local isomorphism in $\operatorname{RelA} \lg (\mathbb{T})$, then $X$ is locally connected provided that $Y$ is locally connected.

Proposition 6.6. The category $\mathbf{L C}_{l i}(\mathbb{T})$ has pullbacks, and they are calculated as in $\mathbf{R e l} \mathbf{A l g}(\mathbb{T})$.

Proof. Follows from Lemma 2.12. 
Proposition 6.7. For every locally connected relational $\mathbb{T}$-algebra $B, \mathbf{L C}_{l i}(\mathbb{T}) / B$ is a full subcategory of $\operatorname{RelAlg}(\mathbb{T}) / B$ closed under colimits.

Proof. Lemma 2.14 shows that the subcategory is full. Lemma 6.2 applies also to $\mathbf{L C}_{l i}(\mathbb{T}) / B$ since coproduct injections are local isomorphisms. It remains to show that $\mathbf{L C}_{l i}(\mathbb{T}) / B$ is closed under coequalizers. Let $f, g: X \rightarrow Y$ be local isomorphisms in $\mathbf{L C}_{l i}(\mathbb{T}) / B$ and $q: Y \rightarrow Q$ their coequalizer in $\operatorname{RelAlg}(\mathbb{T}) / B$ :

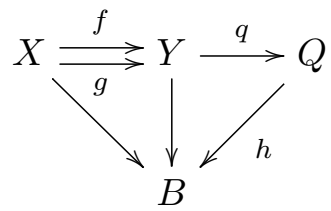

Since the functor $\mathcal{O}$ preserves coequalizers, $\mathcal{O}(q)$ is the coequalizer of the open continuous maps $\mathcal{O}(f), \mathcal{O}(g)$, hence $\mathcal{O}(q)$ is an open surjection, by [2, Lemma 6.4.4]. Hence, using again Lemma 2.14 we can conclude that $h$ is a local isomorphism, and so $q$ is a local isomorphism as well.

In order to study effective descent morphisms in $\mathbf{L C}_{l i}(\mathbb{T}) / B$, first we prove that local isomorphisms are exponentiable in $\operatorname{RelAlg}(\mathbb{T})$. To conclude that we show that the partial product in PsRelAlg $(\mathbb{T})$ of any local isomorphism and any relational $\mathbb{T}$-algebra belongs to $\operatorname{RelAlg}(\mathbb{T})$. We recall that, for any morphism $f:(X, a) \rightarrow(Y, b)$ and any $(Z, c)$ in $\operatorname{PsRelAlg}(\mathbb{T})$, the partial product of $f$ and $(Z, c)$ (see [14])

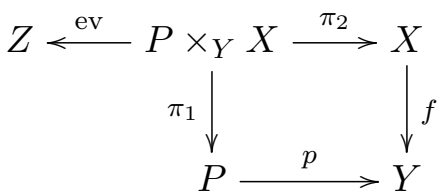

is given by $P=\left\{(s, y) \mid y \in Y, s:\left(f^{-1}(y), \tilde{a}\right) \rightarrow(Z, c)\right.$ in PsRelAlg $\left.(\mathbb{T})\right\}$, where $\tilde{a}$ is the initial structure induced by $a$, with the relational structure defined by

$$
\mathfrak{p} \rightarrow(s, y) \quad \text { if } \quad\left\{\begin{array}{l}
T p(\mathfrak{p}) \rightarrow y \text { and } \\
T \text { ev( }(\mathfrak{w}) \rightarrow \operatorname{ev}((s, y), x)=s(x), \text { whenever } \mathfrak{w} \in T\left(P \times_{Y} X\right) \text { and } x \in X \\
\text { are such that } T \pi_{1}(\mathfrak{w})=\mathfrak{p}, f(x)=y \text { and } T \pi_{2}(\mathfrak{w}) \rightarrow x
\end{array}\right.
$$

for $\mathfrak{p} \in T P$ and $(s, y) \in P$. (For detailed information on this construction see [11].)

Here we need that the natural transformation $\mu: T^{2} \rightarrow T$ has the Beck-Chevalley property $(\mathrm{BC})$, that is, every naturality diagram

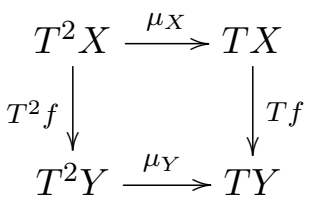

is a (BC)-square, when $f: X \rightarrow Y$ is a map.

Theorem 6.8. If the natural transformation $\mu: T^{2} \rightarrow T$ has (BC), then local isomorphisms are exponentiable in $\operatorname{RelAlg}(\mathbb{T})$.

Proof. Let $f:(X, a) \rightarrow(Y, b)$ be a local isomorphism in $\operatorname{RelAlg}(\mathbb{T})$ and let $(Z, c)$ be a relational $\mathbb{T}$-algebra. Let $(P, d)$ be their partial product in PsRelAlg $(\mathbb{T})$ as defined in $(\mathrm{D})$. We want to show that $(P, d)$ is a relational $\mathbb{T}$-algebra. For that we let $\mathfrak{P} \rightarrow \mathfrak{p} \rightarrow(s, y)$ in $P$, and show that $\mu_{P}(\mathfrak{P}) \rightarrow(s, y)$. Firstly, we observe that $T p\left(\mu_{P}(\mathfrak{P})\right) \rightarrow y=p(s, y)$ since $(Y, b)$ is a relational $\mathbb{T}$-algebra. Secondly, let $\mathfrak{w} \in T\left(P \times_{Y} X\right)$ and $x \in X$ with $T \pi_{1}(\mathfrak{w})=\mu_{P}(\mathfrak{P}), f(x)=y$ and 
$T \pi_{2}(\mathfrak{w}) \rightarrow x$. Let $u: U \rightarrow X$ be an open embedding with $x \in U$ and $U \stackrel{u}{\longrightarrow} X \stackrel{f}{\longrightarrow} Y$ an open embedding. If we form the pullback $u^{\prime}: U^{\prime} \rightarrow P \times_{Y} X$ of $u$ along $\pi_{1}$, both $u^{\prime}$ and $f^{\prime}:=\pi_{2} \cdot u^{\prime}$ are open embeddings. Furthermore:

(a) (BC) of $T$ implies that $\mathfrak{w} \in T U^{\prime}$ since $T \pi_{2}(\mathfrak{w}) \in T U$, because $u$ is open and $T \pi_{2}(\mathfrak{w}) \rightarrow x \in U$.

(b) With $f^{\prime}: U^{\prime} \rightarrow P$, also $T f^{\prime}$ open, and $\mathfrak{P} \rightarrow \mathfrak{p} \rightarrow(s, y)=f^{\prime}(s, x)$ implies that

(i) there exists $\mathfrak{w}^{\prime} \in T U^{\prime}$ such that $T f^{\prime}\left(\mathfrak{w}^{\prime}\right)=\mathfrak{p}$ and $\mathfrak{w}^{\prime} \rightarrow(s, x)$ in $U^{\prime}$;

(ii) there exists $\mathfrak{W} \in T^{2} U^{\prime}$ such that $T^{2} f^{\prime}(\mathfrak{W})=\mathfrak{P}$ and $\mathfrak{W} \rightarrow \mathfrak{w}^{\prime}$.

(c) With $f^{\prime}$, also $T f^{\prime}$ is injective, hence $\mathfrak{w}=\mu_{U^{\prime}}(\mathfrak{W})$ since $T f^{\prime}(\mathfrak{w})=T \pi_{1}(\mathfrak{w})=\mu_{P}(\mathfrak{P})=$ $T f^{\prime}\left(\mu_{U^{\prime}}(\mathfrak{W})\right)$.

Since $(Z, c)$ is a relational $\mathbb{T}$-algebra and $T^{2} \mathrm{ev}(\mathfrak{W}) \rightarrow T$ ev $\left(\mathfrak{w}^{\prime}\right) \rightarrow s(x)$,

$$
\mu_{Z}\left(T^{2} \operatorname{ev}(\mathfrak{W})\right)=T \operatorname{ev}\left(\mu_{P \times_{Y} X}(\mathfrak{W})\right) \rightarrow s(x) .
$$

But this means that $T \mathrm{ev}(\mathfrak{w}) \rightarrow s(x)$ and the conclusion follows.

Theorem 6.9. If the natural transformation $\mu: T^{2} \rightarrow T$ has $(B C)$, then a morphism in $\mathbf{L C}_{l i}(\mathbb{T})$ is of effective descent if, and only if, it is surjective.

Proof. If $p: E \rightarrow B$ is an effective descent morphism in $\mathbf{L C}_{l i}(\mathbb{T})$, then the change of base functor

$$
p^{*}: \mathbf{L C}_{l i}(\mathbb{T}) / B \rightarrow \mathbf{L C}_{l i}(\mathbb{T}) / E
$$

reflects isomorphisms. If we factor $p$ via its image factorization in $\operatorname{RelAlg}(\mathbb{T})$, that is $p=m \cdot p^{\prime}$, with $p^{\prime}$ a surjection and $m$ an embedding, then both $p^{\prime}$ and $m$ are local isomorphisms. But $p^{*}\left(p^{\prime}\right)$ is the identity, hence $p^{\prime}$ is an isomorphism, and therefore $p$ is surjective.

To show the converse, let $p: E \rightarrow B$ be a surjective morphism in $\mathbf{L C}_{l i}(\mathbb{T})$.

(a) $p^{*}$ has a left adjoint, $p$ !, and the counit of the adjunction is pointwise an isomorphism, hence $p^{*}$ is fully faithful; in particular, $p^{*}$ reflects isomorphisms.

(b) $p^{*}: \operatorname{RelAlg}(\mathbb{T}) / B \rightarrow \operatorname{RelAlg}(\mathbb{T}) / E$ has a right adjoint, since $p$ is exponentiable by Theorem 6.8 , hence it preserves colimits. Moreover, in the commutative diagram

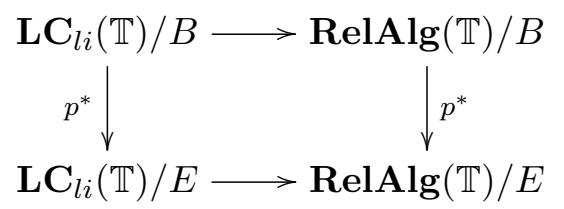

the horizontal embeddings preserve colimits, with $\mathbf{L C}_{l i}(\mathbb{T}) / B$ and $\mathbf{L C}_{l i}(\mathbb{T}) / E$ cocomplete categories, therefore $p^{*}: \mathbf{L C}_{l i}(\mathbb{T}) / B \rightarrow \mathbf{L C}_{l i}(\mathbb{T}) / E$ also preserves colimits.

We can therefore conclude that $p^{*}$ is monadic.

The results of this section show that the situation for a general monad $\mathbb{T}$, satisfying the stated conditions, is very similar to our guiding example Top. A subsequent question - that is not addressed here - is whether it is possible to characterize covering morphisms in Top via ultrafilter convergence, or, in other categories of relational $\mathbb{T}$-algebras, via relational $\mathbb{T}$-algebra structures.

\section{REFERENCES}

[1] M. Barr, Relational algebras, in: Reports of the Midwest Category Seminar, IV, Lecture Notes in Mathematics, Vol. 137. Springer, Berlin, 1970, pp. 39-55.

[2] F. Borceux and G. Janelidze, Galois Theories, Cambridge Studies in Advanced Mathematics, 72, Cambridge Univ. Press 2001. 
[3] C. Cassidy, M. Hébert and G. M. Kelly, Reflective subcategories, localizations and factorization systems. J. Austral. Math. Soc. Ser. A 38 (1985), 287-329.

[4] M. M. Clementino and D. Hofmann, Triquotient maps via ultrafilter convergence, Proc. Amer. Math. Soc. 130 (2002), 3423-3431.

[5] M. M. Clementino and D. Hofmann, Topological features of lax algebras, Appl. Categ. Structures 11 (2003), 267-286.

[6] M. M. Clementino and D. Hofmann, Effective descent morphisms in categories of lax algebras, Appl. Categ. Structures 12 (2004), 413-425.

[7] M. M. Clementino and D. Hofmann, Descent morphisms and a van Kampen Theorem in categories of lax algebras, Topology Appl. 159 (2012), 2310-2319.

[8] M. M. Clementino, D. Hofmann and G. Janelidze, Local homeomorphisms via ultrafilter convergence, Proc. Amer. Math. Soc. 133 (2005), 917-922.

[9] M. M. Clementino, D. Hofmann and G. Janelidze, On exponentiability of étale algebraic homomorphisms, J. Pure Appl. Algebra 217 (2013), 1195-1207.

[10] M. M. Clementino, D. Hofmann and G. Janelidze, The monads of classical algebra are seldom weakly cartesian, DMUC Preprint 12-46, University of Coimbra 2012.

[11] M. M. Clementino, D. Hofmann and W. Tholen, Exponentiability in categories of lax algebras, Theory Appl. Categories 11 (2003), 337-352.

[12] M. M. Clementino and G. Janelidze, A note on effective descent morphisms of topological spaces and relational algebras, Topology Appl. 158 (2011), 2431-2436.

[13] M. M. Clementino and W. Tholen, Metric, topology and multicategory - a common approach, J. Pure Appl. Algebra 179 (2003), 13-47.

[14] R. Dyckhoff and W. Tholen, Exponentiable morphisms, partial products and pullback complements, J. Pure Appl. Algebra 49 (1987), 103-116.

[15] G. Janelidze, The fundamental theorem of Galois theory, Math USSR Sbornik 64 (1989), 359-374.

[16] G. Janelidze, Pure Galois theory in categories, J. Algebra 132 (1990), 270-286.

[17] G. Janelidze, Categorical Galois theory: Revision and some recent developments, in: Galois connections and applications, pp. 139-171, Math. Appl., 565, Kluwer Acad. Publ., Dordrecht, 2004.

[18] G. Janelidze, Descent and Galois Theory, Lecture Notes of the Summer School in Categorical Methods in Algebra and Topology, Haute Bodeux, Belgium, 2007 (available at http://www.math.yorku.ca/ tholen/).

[19] G. Janelidze and W. Tholen, How algebraic is the change of base functor?, in: Springer Lect. Notes in Math. 1488 (1991), 174-186.

[20] G. Janelidze and M. Sobral, Finite preorders and topological descent I, J. Pure Appl. Algebra 175 (2002), 187-205.

[21] M. Mahmoudi, C. Schubert and W. Tholen, Universality of coproducts in categories of lax algebras, Appl. Categ. Structures 14 (2006), 243-249.

[22] E. Manes, Taut monads and T0-spaces, Theoret. Comput. Sci. 275 (2002), 79-109.

[23] J. Reiterman and W. Tholen, Effective descent maps of topological spaces, Top. Appl. 57 (1994), 53-69.

[24] J. J. Xarez, The monotone-light factorization for categories via preordered and ordered sets, $\mathrm{PhD} \mathrm{Thesis,}$ Univ. Aveiro, 2003.

CmuC, Department of Mathematics, University of Coimbra, 3001-454 Coimbra, Portugal E-mail address: mmc@mat.uc.pt

Cidma, Departamento de Matemática, Universidade de Aveiro, 3810-193 Aveiro, Portugal E-mail address: dirk@ua.pt

CMuC, Department of Mathematics, University of Coimbra, 3001-454 Coimbra, Portugal

E-mail address: montoli@mat.uc.pt 